\title{
DISPONIBILIDADE HÍDRICA DA BACIA DO RIO PIRACICABA, ESTADO DE SÃO PAULO, BRASIL
}

\section{AVAILABILITY OF WATER RESOURCES OF PIRACICABA RIVER BASIN, SAO PAULO STATE, BRAZIL}

\section{Pereira,N.M. ${ }^{1}$, Pereira, S.Y. ${ }^{1}$, Cavalcanti, R.N. ${ }^{1}$, Genovez, A.M.'; Paula, G. ${ }^{1}$}

${ }^{1}$ Instituto de Geociências - UNICAMP, CP 6152, CEP 13083-970, Campinas, São Paulo, Brasil.

${ }^{2}$ Faculdade de Engenharia Civil - UNICAMP, CP 6021, CEP 13083-971, Campinas, São Paulo, Brasil.

\section{RESUMO}

O presente artigo visa avaliar a disponibilidade hídrica na bacia do rio Piracicaba, confrontando dados de consumo de água, atual e futuro com as análises de vazões (média mínima e máxima mensais) do monitoramento existente do DAEE/ CTH. Um extenso trabalho de análise das informações existentes sobre o consumo e perfil de usuários foi realizado, em publicações existentes (artigos científicos, relatórios de situação e planos de recursos hídricos) e atualizados por meio em órgãos de controle e consultas diretas com o usuário. A bacia do rio Piracicaba compreende uma área de drenagem de $12.400 \mathrm{Km}^{2}, 90 \%$ dos quais situados no estado de São Paulo e o restante no extremo Sul de Minas Gerais. A população residente na bacia do rio Piracicaba totaliza 3,1 milhões de pessoas (SEADE,1998). A disponibilidade hídrica média na bacia é de $165 \mathrm{~m}^{3} / \mathrm{s}$, caindo para 40 $\mathrm{m}^{3} / \mathrm{s}$ em épocas de estiagens. O Sistema Cantareira, que transpõe as águas da bacia para a Região Metropolitana de São Paulo, não tem mantido a vazão mínima de $40 \mathrm{~m}^{3} / \mathrm{s}$ estabelecida para o rio Piracicaba. $\mathrm{O}$ uso urbano $\left(11,2 \mathrm{~m}^{3} / \mathrm{s}\right)$ e o industrial $\left(9,9 \mathrm{~m}^{3} / \mathrm{s}\right)$ são responsáveis pela maior parte do consumo de água na Bacia (81\%) enquanto o uso para a irrigação é responsável por $19 \%\left(25 \mathrm{~m}^{3} / \mathrm{s}\right)$. Há uma tendência de estabilização do consumo industrial e o crescimento do consumo urbano ( $2 \%$ a.a.) e do agropecuário ( $1,34 \%$ a.a.), totalizando um aumento de demanda de 0,35 $\mathrm{m}^{3} / \mathrm{s}$ a.a. O balanço das vazões superficiais indica que a Sub-Bacia do rio Jaguari possui as maiores vazões disponíveis, de 23,4 m³ $/ \mathrm{s}$. Em apenas 13\% 
do ano essa Sub-Bacia não dispõe, para manter a vazão de $40 \mathrm{~m}^{3} / \mathrm{s}$ no rio Piracicaba junto à cidade homônima, de vazões para atender a demanda prevista.

Palavras chaves: recurso hídrico, disponibilidade, bacia hidrográfica, rio Piracicaba, demanda.

\begin{abstract}
The aim of this paper is to evaluate the water availability of the Piracicaba river basin, comparing water consumption data, both actual and future, with the analyses of outflows (monthly average, minimum and maximum figures) from the existing $\mathrm{DAEE} / \mathrm{CTH}$ monitoring program. Extensive analysis was carried out of existing information, both in relation to consumption as well as of user profile, in scientific publications, technical reports, and water availability plans) and the information was brought up-to-date with the help of the relevant government regulatory bodies and interviews with consumers. The Piracicaba river basin has a drainage area of $12,400 \mathrm{Km}^{2}$, of which $90 \%$ is situated in the State of Sao Paulo, with the remaining $10 \%$ being in the States of Minas Gerais and Rio de Janeiro. The Piracicaba river basin's population comes to a total of 3.1 million people (SEADE 1998). The average water availability in the basin is $165 \mathrm{~m}^{3} / \mathrm{s}$, dropping to $40 \mathrm{~m}^{3} / \mathrm{s}$ in periods of drought. The Cantareira System, which carries the waters of the basin to Sao Paulo's Metropolitan Region, has not been maintaining the minimum outflow $\left(40 \mathrm{~m}^{3} / \mathrm{s}\right)$ established for the Piracicaba River. Urban usage $\left(11.2 \mathrm{~m}^{3} / \mathrm{s}\right)$ and industrial usage $\left(9.9 \mathrm{~m}^{3} / \mathrm{s}\right)$ account for $81 \%$ of the water consumption in the Piracicaba Basin, while usage for irrigation purposes represents a further $19 \%\left(25 \mathrm{~m}^{3} / \mathrm{s}\right)$. There is a trend towards stabilization in terms of industrial consumption and growth in urban consumption $(+2 \%$ per year) and in consumption for irrigation purposes $(+1.34 \%$ per year), resulting in a yearly increase in demand of $0.35 \mathrm{~m}^{3} / \mathrm{s}$. An examination of river surface discharge levels suggests that the Jaguari river sub basin has the highest discharge values available, $23.4 \mathrm{~m}^{3} / \mathrm{s}$. During just $13 \%$ of the year, this river does not have a sufficient level of discharge to maintain an outflow rate of $40 \mathrm{~m}^{3} / \mathrm{s}$ in the Piracicaba river, near the city bearing the same name.
\end{abstract}

Key words: water resources, availability, watershed, Piracicaba river, demand. 


\section{INTRODUÇÃO}

O presente artigo trata da disponibilidade hídrica na bacia do rio Piracicaba, confrontando dados de consumo de água, atual e futuro, captada nessa Bacia com dados de vazões monitoradas por órgãos que se ocupam da sua gestão.

A bacia do rio Piracicaba compreende uma área de drenagem de $12.600 \mathrm{Km}^{2}, 90 \%$ dos quais situados no estado de São Paulo e o restante no extremo Sul de Minas Gerais. A localização, a extensão territorial e o volume de seus recursos hídricos foram, sem sombra de dúvida, elementos decisivos para que hoje a região de influência da Bacia possa ser caracterizada como uma das mais ricas do país.

A Bacia se subdivide em quatro sub-bacias principais, a sub-bacia do Piracicaba com $3770 \mathrm{~km}^{2}$, a do Jaguari com $2180 \mathrm{~km}^{2}$, a do Atibaia com $2820 \mathrm{~km}^{2}$ e a sub-bacia do Corumbataí com seus $1690 \mathrm{~km}^{2}$ de área drenada. Afora essas sub-bacias, convergem para ela as águas dos rios Camanducaia e Passa Cinco, e dos ribeirões Anhumas, Pinheiros, Quilombo, Lava-Pés, dos Toledos, Tijuco Preto, Tatu e Claro.

$\mathrm{Na}$ área da Bacia situa-se um dos principais parques industriais do país, resultado de um processo de interiorização industrial iniciado na década de 70, que se fez acompanhar por intensa urbanização hoje refletida numa malha de 45 cidades dotadas de boa infra-estrutura. Situa-se, ainda, extensa atividade agrícola sucro-alcooleira, de cítricos e de produtos hortifrutigranjeiros.

A população residente totaliza 3,1 milhões de pessoas (SEADE,1998) concentrada principalmente nas sub-bacias dos rios Piracicaba e Atibaia, cada qual com cerca de $40 \%$ da população total da Bacia. As sub-bacias dos rios Jaguari e Corumbataí perfazem, respectivamente, apenas $13 \%$ e $6 \%$ dessa população total.

Não obstante a intensidade da atividade produtiva e a concentração populacional existente na área de influência da Bacia, ela ainda suporta a transposição de suas águas pelo Sistema Cantareira para abastecer a Região Metropolitana de São Paulo à vazão média de $31 \mathrm{~m}^{3} / \mathrm{s}$, captação que se concentra nas sub-bacias dos rios Atibaia e Jaguari. O Sistema Cantareira é formado pela captação dos rios Jaguari, Cachoeira e Atibainha, que formam o rio Piracicaba e o Jaguari, fora da bacia.

Os múltiplos usos das águas, que a Bacia proporciona, tornam-na complexa para análise, situação que se agrava devido a inexistência de registros históricos confiáveis, muitas vezes inconsistentes quando diferentes fontes são consultadas (PELLEGRINO, 1995).

A precipitação média anual em toda a bacia do Piracicaba é de aproximadamente $1400 \mathrm{~mm}$, com máximas nos meses de dezembro e 
janeiro e mínimas em agosto.

A vazão natural média é da ordem de $185 \mathrm{~m}^{3} / \mathrm{s}$, correspondendo a $465 \mathrm{~mm}$ de precipitação, de modo que apenas 33\% da água de precipitação são convertidos em escoamento superficial.

\section{PERFIL DE CONSUMO DOS RECURSOS HÍDRICOS NA BACIA}

Os relatórios e trabalhos que enfocam o consumo d'água na bacia do rio Piracicaba apresentam dados nem sempre compatíveis entre si ou que possam ser considerados absolutamente confiáveis, razão pela qual é necessário recorrer com freqüência à conferência das informações obtidas através de uma fonte com outras. Dificuldades à parte, resulta que não há uma literatura guia de onde dados de consumo e usos possam ser obtidos mas, sim, um conjunto de publicações que, ora uma ora outra, apresentam informações úteis ao tipo de investigação proposto.

Antes que se passe a outras considerações, é importante informar que os dados a respeito do consumo vão se referir, sempre que não for devidamente explicitado, ao volume de captação e não propriamente ao uso consuntivo, para o qual a confiabilidade das informações, pode-se afirmar, é precaríssima.

Além de consulta direta às empresas, os dados aqui tratados foram obtidos junto à Secretaria do Meio Ambiente (SMA), ao Departamento de Água e Energia Elétrica (DAEE), ao Comitê das Bacias Hidrográficas dos Rios Piracicaba, Capivari e Jundiaí (CBH-PCJ) e a Fundação Sistema Estadual de Análise de Dados (SEADE).

A bacia do rio Piracicaba, excluída a captação pelo Sistema Cantareira, sustenta um volume de captação de água de $25,31 \mathrm{~m}^{3} / \mathrm{s}$, distribuídos por suas sub-bacias conforme apresentado na Tabela 01 . Expressando os dados dessa Tabela em percentuais fica claro que o uso urbano e o industrial são responsáveis pela maior parte do consumo de água na Bacia como um todo (81\%), enquanto o uso na irrigação é responsável por apenas $19 \%$. É bom que se ressalte que o consumo para irrigação contempla o setor agropecuário sem o consumo correspondente às usinas sucro-alcooleiras, computado junto ao setor industrial.

Tabela 01. Consumo de Água na Bacia do Rio Piracicaba - 1994/1995 (m³/s)

\begin{tabular}{|l|c|c|c|c|c|c|c|c|c|}
\hline \multirow{2}{*}{$\begin{array}{l}\text { SUB- } \\
\text { BACIA }\end{array}$} & \multicolumn{2}{|c|}{ INDUSTRIAL } & \multicolumn{2}{c|}{ URBANO } & \multicolumn{2}{c|}{ IRRIGAÇÃO } & \multicolumn{2}{c|}{ TOTAL POR SUB-BACIA } \\
\cline { 2 - 10 } & 1994 & 1995 & 1994 & 1995 & 1994 & 1995 & 1994 & 1995 & Variação \\
\hline Atibaia & 2,71 & 2,84 & 4,49 & 4,55 & 1,44 & 1,45 & 8,64 & 8,84 & $2,31 \%$ \\
\hline Corumbataí & 0,78 & 0,89 & 1,36 & 1,52 & 0,37 & 0,38 & 2,51 & 2,79 & $11,16 \%$ \\
\hline Jaguari & 2,53 & 2,51 & 1,94 & 1,91 & 1,83 & 1,85 & 6,3 & 6,27 & $-0,48 \%$ \\
\hline Piracicaba & 3,95 & 3,73 & 2,27 & 2,52 & 1,15 & 1,16 & 7,37 & 7,41 & $0,54 \%$ \\
\hline Total & 9,97 & 9,97 & 10,06 & 10,5 & 4,79 & 4,84 & 24,82 & 25,31 & $1,97 \%$ \\
\hline
\end{tabular}

Fonte: Relatório de Situação dos Recursos Hídricos, 1995 / СBH/PCJ, 1996. 
Uso industrial - $\mathrm{O}$ consumo de água para fins industriais em toda a bacia do Piracicaba manteve-se constante nos anos de 1994 e 1995, alcançando um volume de $9,97 \mathrm{~m}^{3} / \mathrm{s}$ conforme consta na Tabela 01 . Para conhecer o comportamento retrospectivo do consumo por períodos mais longos, contudo, é necessário lançar mão de fontes diversas, agrupadas na Tabela 02. Nessa mesma Tabela também são apresentados os dados de captação, entre 1984 e 1998, de 17 empresas instaladas na Bacia que foram objeto de consulta específica, as quais, em seu conjunto, utilizam um volume de recursos hídricos correspondente a $90 \%$ do volume total nela captado.

Quando são analisados volumes de captação fornecidos na (Tabela 02) conclui-se que o consumo de 9 das 17 empresas consultadas mantevese constante nos últimos 5 anos (1993-1998), empresas essas que respondem por $52 \%$ do total de água para fins industriais subtraída da Bacia, ou seja, $5 \mathrm{~m}^{3} / \mathrm{s}$. Por outro lado, apenas duas empresas aumentaram os volumes captados e uma diminuiu seu consumo. Para as 5 restantes, o CBH-PCJ não dispõe de informações.

Complementando-se as informações do CBH-PCJ com as fornecidas por (Monticeli e Martins, 1993) conseguem-se dados de captação do período de 1992 a 1998 para 16 das 17 empresas consultadas. Esses permitem concluir que 8 delas diminuíram seus volumes captados, 5 mantiveram os volumes constantes e apenas 3 empresas aumentaram a captação nos seis anos investigados .

Quando se trata com projeções de consumo industrial na Bacia, verifica-se que as elaboradas por (SÃO PAULO, (SMA), 1994) apontam taxas de crescimento de $1,2 \%$ a.a. para a década de 90 e de $4,2 \%$ a.a. para a década seguinte, o que resultaria num consumo de $14,7 \mathrm{~m}^{3} / \mathrm{s}$ no final da década de 90 e de $21,33 \mathrm{~m}^{3} / \mathrm{s}$ em 2010. Tais projeções revelam-se, atualmente, superestimadas, uma vez que em 1998 o consumo de água para uso industrial alcançou tão somente $9,44 \mathrm{~m}^{3} / \mathrm{s}$, segundo informação do CBH-PCJ, volume significativamente inferior ao prognosticado.

Do exposto não é difícil concluir que não há aumento do consumo industrial na bacia do rio Piracicaba desde o início da década de 90, afirmação que também pode ser conferida através das concessões de outorga para captação de água no Estado, fornecidas pelo DAEE, as quais indicam um volume de captação de $9,58 \mathrm{~m}^{3} / \mathrm{s}$.

Se os dados apresentados são contundentes para afirmar que na década de 90 o aumento da captação pelo setor industrial é nulo, a cobrança pelo uso dos recursos hídricos, cuja lei deverá ser homologada, poderá ser outro fator que poderá manter essa tendência. 
Tabela 02 - Uso Industrial segundo fontes e datas diversas

\begin{tabular}{|c|c|c|c|c|c|c|c|c|c|c|c|c|}
\hline \multirow{4}{*}{ EMPRESAS } & \multirow{4}{*}{$\begin{array}{c}\text { SUB-BACIA } \\
\text { DE } \\
\text { CAPTAÇÃO }\end{array}$} & \multicolumn{11}{|c|}{ CAPTAÇÃO $\left(\mathrm{M}^{3} / \mathrm{S}\right)$ E DESPEJO } \\
\hline & & \multirow{3}{*}{\begin{tabular}{|l|}
1984 \\
$\begin{array}{l}\text { São Paulo } \\
1994\end{array}$ \\
Captação \\
\end{tabular}} & \multirow{2}{*}{\multicolumn{2}{|c|}{$\begin{array}{c}1992 \\
\text { Monticeli e } \\
\text { Martins. } 1993\end{array}$}} & \multirow{3}{*}{\begin{tabular}{|c|}
1993 \\
CBH- \\
PCJ. \\
1994 \\
Captação \\
\end{tabular}} & \multicolumn{7}{|c|}{1998} \\
\hline & & & & & & \multicolumn{2}{|c|}{ Consulta Empresas } & \multicolumn{2}{|c|}{ СВН-РCЈ } & \multirow{2}{*}{\begin{tabular}{|c|} 
SMA \\
Captação \\
\end{tabular}} & \multicolumn{2}{|l|}{ DAEE } \\
\hline & & & Captação & Despejo & & Captação & Despejo & Captação & Despejo & & Captação & Despejo \\
\hline Rhodia Indústria Química & Atibaia & 4,00 & 2,92 & 2,78 & 2,04 & 2,08 & 2,01 & 2,04 & 1,87 & 2,92 & 2,04 & 1,87 \\
\hline Shell Química S.A. & Atibaia & & 0,17 & 0,17 & 0,17 & 0,06 & 0,06 & 0,17 & 0,17 & 0,17 & 0,17 & 0,17 \\
\hline J. Bresler S.A. Ind. De Papel & Atibaia & & 0,17 & 0,14 & 0,17 & 0,07 & 0,07 & 0,17 & 0,14 & 0,17 & 0,17 & 0,14 \\
\hline Sub-Total & Atibaia & 4,00 & 3,25 & 3,09 & 2,38 & 2,22 & 2,14 & 2,37 & 2,18 & 3,26 & 2,37 & 2,18 \\
\hline Usina Costa Pinto S.A. & Corumbataí & 0,39 & 0,47 & 0,19 & 0,28 & & & 0,28 & 0,14 & & 0,28 & 0,14 \\
\hline S.A Ind. Química Butilamil & Corumbataí & & 0,21 & 0,18 & 0,21 & 0,28 & 0,27 & 0,46 & 0,46 & 0,21 & 0,46 & 0,46 \\
\hline Santa Luzia S.A./ Salusa & Corumbataí & & & & & & & 0,01 & 0,01 & & 0,01 & 0,01 \\
\hline Sub-total & Corumbataí & 0,39 & 0,68 & 0,37 & 0,49 & 0,28 & 0,27 & 0,75 & 0,61 & 0,21 & 0,75 & 0,61 \\
\hline Ajinomoto Interamericana & Jaguari & 0,31 & 1,16 & 1,04 & 0,35 & 0,50 & 0,40 & 0,35 & 0,33 & 1,16 & 0,35 & 0,33 \\
\hline Papirus Ind. De Papel S.A. & Jaguari & 0,14 & 0,23 & 0,20 & 0,14 & & & 0,14 & 0,13 & 1,14 & 0,14 & 0,13 \\
\hline Fáb. de Papel Santa Therezinha & Jaguari & & 0,12 & 0,06 & & 0,07 & 0,05 & 0,09 & 0,07 & 0,12 & 0,09 & 0,07 \\
\hline Petrobrás REPLAN & Jaguari & 0,34 & 0,56 & 0,34 & 0,40 & 0,39 & 0,17 & 0,44 & & 0,56 & 0,44 & \\
\hline Usina Açucareira Ester S.A & Jaguari & & 1,08 & 0,89 & 0,56 & & & 3,34 & 3,33 & & 3,34 & 3,33 \\
\hline Cia. Antarctica Paulista & Jaguari & & 0,11 & & & 0,17 & 0,14 & 0,11 & 0,09 & & 0,11 & 0,09 \\
\hline Sub-total & Jaguari & 0,79 & 3,26 & 2,53 & 1,45 & 1,12 & 0,76 & 4,47 & 3,95 & 2,98 & 4,47 & 3,95 \\
\hline Ripasa S.A. Celulose e Papel & Piracicaba & 0,72 & 1,00 & 0,89 & 1,00 & & & 1,00 & 0,64 & & 1,00 & 0,64 \\
\hline Limeira S.A. Ind. De Papel e Cart. & Piracicaba & & 0,19 & 0,21 & & & & 0,11 & 0,11 & 0,11 & 0,11 & 0,11 \\
\hline Fibra S.A. & Piracicaba & & 0,32 & 0,31 & 0,32 & & & 0,32 & 0,31 & 0,32 & 0,32 & 0,31 \\
\hline Usina São José S.A. & Piracicaba & & 0,26 & 0,20 & 0,30 & 0,26 & 0,20 & 0,16 & 0,12 & & 0,16 & 0,12 \\
\hline Cia. Ind. Agr. Ometto/ Us.Iracema & Piracicaba & 0,47 & 0,05 & 0,00 & & 0,22 & 0,00 & 0,26 & 0,08 & & 0,40 & \\
\hline Sub-total & Piracicaba & 1,19 & 1,81 & 1,61 & 1,62 & 0,48 & 0,20 & 1,85 & 1,27 & 0,43 & 1,99 & 1,19 \\
\hline TOTAL & & 6,38 & 9,00 & 7,59 & 5,94 & 4,09 & 3,38 & 9,44 & 8,00 & 6,88 & 9,58 & 7,92 \\
\hline
\end{tabular}

Fonte: Elaboração própria com base nas informações bibliográficas existentes e nas consultas realizadas em SÃO PAULO (1994 e 1998), Monticeli e Martins (1993), CBH-PCJ (1994 e 1998), DAEE (1998) 
A instituição da cobrança certamente fomentará, num primeiro momento, a adoção de medidas contra o desperdício para, em seguida, estimular a implantação de sistemas de gestão ambiental (SGA). Por outro lado, a arrecadação de tributo pelo uso da água terá impactos que indiretamente levarão à racionalidade de uso do recurso, a exemplo de uma efetiva fiscalização e fomento às boas práticas.

Uso urbano - $\mathrm{O}$ consumo urbano de recursos hídricos na bacia do rio Piracicaba é de $11,2 \mathrm{~m}^{3} / \mathrm{s}$, como não poderia deixar de ser, fortemente influenciado pela concentração populacional existente em cada uma das sub-bacias que a constituem.

A Tabela 03 apresenta uma síntese da evolução do crescimento da população residente na Bacia assim como de sua urbanização. Dela se depreende que as maiores taxas de crescimento populacional verificam-se na sub-bacia do rio Piracicaba (2,44\% a.a.), a qual supera em $0,43 \%$ a média registrada para a totalidade da Bacia (2,01\% a.a.). Nessa Sub-Bacia, os municípios de Hortolândia e Sumaré apresentam as maiores taxas de crescimento populacional e, por conseguinte, enfrentam as maiores restrições quanto a disponibilidade de água para abastecimento urbano da região.

A partir de informações fornecidas pelo SEADE sobre o crescimento populacional nos anos de 1996 e 1997 projetou-se a expectativa de evolução da população da bacia do rio Piracicaba (Tabela 04), podendo-se prognosticar que:

- devido ao crescimento populacional diferenciado, a sub-bacia do rio Piracicaba deve aumentar a sua participação relativa na população da Bacia como um todo para cerca de $43 \%$ em 2005, continuando a crescer à taxas superiores à média da Bacia. Ao contrário, a participação relativa na população da sub-bacia do rio Atibaia deve diminuir para $38 \%$;

- as participações relativas das populações das sub-bacias do Corumbataí e do Jaguari devem permanecer estáveis;

- o consumo de água para fins urbanos na bacia do Piracicaba deve aumentar em aproximadamente $49 \%$ entre 2000 e 2020, passando assim de 11,2 para $16,8 \mathrm{~m}^{3} / \mathrm{s}$. Esse aumento será diferenciado no tempo, estimando-se em $10 \%$ até 2005 e em $22 \%$ até 2010 .

De imediato é possível concluir que a maior taxa de crescimento populacional apresentada pelos municípios da sub-bacia do rio Piracicaba $(2,44 \%)$ implica em crescente uso e consumo de água para abastecimento urbano que, por sua vez, se depara com dois fatores limitantes: disponibilidade e poluição.

A exemplo do que já acontece na cidade de Piracicaba e em municípios vizinhos, o crescimento da população e da poluição tem levado 
a não disponibilidade de água superficial, estimulando a procura de alternativas de manancial para suprimento urbano. O rio Jaguari, menos poluído, com menor população urbana, com população apresentando tendência à estabilidade de crescimento, se apresenta como a alternativa mais promissora.

Ao que tudo indica, o grande problema a ser enfrentado pelos municípios da bacia do Piracicaba como um todo é, e continuará sendo, o abastecimento urbano, que pressionado pelo crescimento populacional se obriga captar $0,29 \mathrm{~m}^{3} / \mathrm{s}$ adicionais a cada ano.

Tabela 03. Evolução da População e Urbanização na Bacia entre 1996-1997

\begin{tabular}{|l|c|c|c|}
\hline \multicolumn{1}{|c|}{ Sub-bacia } & $\begin{array}{c}\text { Taxa de } \\
\text { Crescimento } \\
\text { Populacional } \\
\mathbf{( \% )}\end{array}$ & $\begin{array}{c}\text { Taxa de } \\
\text { Urbanização } \\
\mathbf{( \% )}\end{array}$ & Características Relevantes \\
\hline Piracicaba & 2,44 & 94,60 & $\begin{array}{c}\text { alta TCP, sendo um dos fatores } \\
\text { limitantes da disponibilidade de água }\end{array}$ \\
\hline Atibaia & 1,50 & 94,14 & $\begin{array}{c}\text { menor TCP, por conta de sua diminuição } \\
\text { no município de Campinas }\end{array}$ \\
\hline Corumbataí & 1,95 & 94,89 & $\begin{array}{c}\text { Municípios de pequeno porte, consumo } \\
\text { urbano baixo }\end{array}$ \\
\hline Jaguari & 2,26 & 83,84 & $\begin{array}{c}\text { população reduzida, não produzindo } \\
\text { impacto significativo no consumo }\end{array}$ \\
\hline Média & 2,01 & 93,03 & \multicolumn{2}{c}{}
\end{tabular}

Fonte: Elaboração própria com base em dados fornecidos pelo SEADE

Tabela 04. Projeções da Demanda de Água para Abastecimento Urbano na Bacia do Piracicaba (2000 - 2020)

\begin{tabular}{|l|c|c|c|c|c|}
\cline { 2 - 6 } \multicolumn{1}{c|}{} & \multicolumn{2}{|c|}{ Demanda em $\mathbf{~ m}^{\mathbf{3}} / \mathbf{s}$} & $\mathbf{b} / \mathbf{a}$ & $\begin{array}{c}\text { Crescimento } \\
\text { anual }\end{array}$ & $\begin{array}{c}\text { Crescimento } \\
\text { médio anual }\end{array}$ \\
\cline { 2 - 6 } & $\mathbf{2 0 0 0}(\mathbf{a})$ & $\mathbf{2 0 2 0}(\mathbf{b})$ & $\mathbf{\%}$ & $\mathbf{\%}$ & $\mathbf{m}^{\mathbf{3}} / \mathbf{s}$ \\
\hline Atibaia & 4,5 & 6,0 & 35 & 1,5 & 0,08 \\
\hline Corumbataí & 0,6 & 0,9 & 48 & 1,9 & 0,02 \\
\hline Jaguari & 1,3 & 2,1 & 56 & 2,2 & 0,04 \\
\hline Piracicaba & 4,8 & 7,8 & 62 & 2,4 & 0,15 \\
\hline Total da Bacia & 11,2 & 16,8 & 50 & 2,0 & 0,29 \\
\hline
\end{tabular}

Fonte: Elaboração própria com base em dados fornecidos pelo SEADE (1998) e CBH-PCJ (1998).

Uso agropecuário - $\mathrm{O}$ uso dos recursos hídricos na agropecuária é fundamentalmente decorrente da captação para irrigação, a qual utilizou em 1995 , tomando-se a Bacia em sua totalidade, um volume de $4,84 \mathrm{~m}^{3} / \mathrm{s}$ (Tabela 01).

Enquanto estudo realizado pela (HIDROPLAN/DAEE, 1994) indica uma tendência de crescimento anual do consumo agropecuário de $0,8 \%$, 
outro estudo publicado pelo (CBH-PCJ, 1996) mostra que a captação para irrigação tem se mantido constante nas sub-bacias que constituem a bacia do rio Piracicaba nos anos de 1994 e 1995.

Por seu turno, estudo coordenado por (GUEDA, STURION e CARON, 1992) projeta para 2010, na bacia do Piracicaba, um uso consuntivo na agropecuária de $13,4 \mathrm{~m}^{3} / \mathrm{s}$. Ao se subtrair desse volume estimado por (GUEDA, STURION e CARON, 1992) o volume de água correspondente ao uso consuntivo das usinas sucro-alcooleiras (fertirrigação), sua estimativa de uso consuntivo total cai para $10,5 \mathrm{~m}^{3} / \mathrm{s}$.

Publicação da Secretaria do Meio Ambiente de 1994 estima o consumo futuro de água por parte do setor agropecuário indicando:

- aumento do consumo, entre 1990 e 2000 , de $20,4 \%$ (de 3,68 para 4,43 $\mathrm{m}^{3} / \mathrm{s}$ ) na Bacia como um todo, o que significa uma taxa anual de crescimento de $1,87 \%$;

- aumento do consumo, entre 2000 e 2010 , de $35,6 \%$ (de 4,43 para 6,01 $\mathrm{m}^{3} / \mathrm{s}$ ) na Bacia como um todo, o que resulta em uma taxa anual de $3,1 \%$;

- aumento do consumo, entre 1990 e 2000 , de $17 \%$ (1,6\% a.a.) na subbacia do Atibaia; de 26,5\% (2,4\%a.a.) na sub-bacia do Jaguari; e de $17 \%$ (1,6\% a.a.) nas sub-bacias do Piracicaba e do Corumbataí juntas;

- aumento do consumo, entre 2000 e 2010 , de $22 \%$ ( $2 \%$ a.a.) na sub-bacia do Atibaia; de 48\% (4\%a.a.) na sub-bacia do Jaguari; de 17\% (3,4\% a.a.) nas sub-bacias do Piracicaba e do Corumbataí juntas.

O que se conclui das estimativas da SMA é a tendência de aumento do uso da água para fins agropecuários na Bacia e que o maior aumento deverá ocorrer na sub-bacia do rio Jaguari, manancial para o qual converge a maioria dos interesses de captação na região.

Mais do que tudo, a divergência entre as estimativas evidencia a precariedade dos dados utilizados na elaboração dos prognósticos sobre a evolução do uso do recurso hídrico no setor agropecuário, que tanto pode sofrer um ligeiro aumento no consumo como tender a estabilização. De qualquer maneira, para contemplar a segurança nos cálculos a serem realizados, optou-se por adotar a taxa de crescimento de consumo do setor agropecuário indicados pelo $\mathrm{CBH} / \mathrm{PCJ}$, ou seja, $1,97 \%$ a.a. (ou $0,06 \mathrm{~m}^{3} / \mathrm{s}$ por ano).

$\mathrm{Na}$ Tabela 05, as tendências indicam, para a Bacia em seu todo, a estabilização dos volumes de captação industrial, o crescimento do consumo urbano à taxa de $2 \%$ a.a. e do agropecuário em $1,1 \%$ a.a.. Percentuais que sinalizam um aumento de demanda total de água de aproximadamente $0,35 \mathrm{~m}^{3} / \mathrm{s}$ a.a. 
Tabela 05. Projeção do Crescimento Médio Anual da Demanda de Água de 2000 a 2020

\begin{tabular}{|c|c|c|c|c|c|}
\hline & Jaguari & Atibaia & Piracicaba & Corumbataí & Total \\
\hline Industrial & $\begin{array}{l}0,0 \% \text { a.a. } \\
0,0 \mathrm{~m}^{3} / \mathrm{s}\end{array}$ & $\begin{array}{l}0,0 \% \text { a.a. } \\
0,0 \mathrm{~m}^{3} / \mathrm{s}\end{array}$ & $\begin{array}{l}0,0 \% \text { a.a. } \\
0,0 \mathrm{~m}^{3} / \mathrm{s}\end{array}$ & $\begin{array}{l}0,0 \% \text { a.a. } \\
0,0 \mathrm{~m}^{3} / \mathrm{s}\end{array}$ & $\begin{array}{l}0,0 \% \text { a.a. } \\
0,0 \mathrm{~m}^{3} / \mathrm{s}\end{array}$ \\
\hline Urbano & $\begin{array}{l}2,25 \% \text { a.a. } \\
0,04 \mathrm{~m}^{3} / \mathrm{s}\end{array}$ & $\begin{array}{l}1,15 \% \text { a.a. } \\
0,08 \mathrm{~m}^{3} / \mathrm{s}\end{array}$ & $\begin{array}{l}2,44 \% \text { a.a. } \\
0,15 \mathrm{~m}^{3} / \mathrm{s}\end{array}$ & $\begin{array}{l}1,9 \% \text { a.a. } \\
0,02 \mathrm{~m}^{3} / \mathrm{s}\end{array}$ & $\begin{array}{l}2,02 \% \text { a.a. } \\
0,29 \mathrm{~m}^{3} / \mathrm{s}\end{array}$ \\
\hline Agropecuário & $\begin{array}{l}1,09 \% \text { a.a. } \\
0,02 \mathrm{~m}^{3} / \mathrm{s}\end{array}$ & $\begin{array}{l}0,69 \% \text { a.a. } \\
0,01 \mathrm{~m}^{3} / \mathrm{s}\end{array}$ & $\begin{array}{l}0,87 \% \text { a.a. } \\
0,01 \mathrm{~m}^{3} / \mathrm{s}\end{array}$ & $\begin{array}{l}2,7 \% \text { a.a. } \\
0,02 \mathrm{~m}^{3} / \mathrm{s}\end{array}$ & $\begin{array}{l}1,34 \% \text { a.a. } \\
0,06 \mathrm{~m}^{3} / \mathrm{s}\end{array}$ \\
\hline $\begin{array}{l}\text { Crescimento } \\
\text { médio anual }\end{array}$ & $0,06 \mathrm{~m}^{3} / \mathrm{s}$ & $0,09 \mathrm{~m}^{3} / \mathrm{s}$ & $0,16 \mathrm{~m}^{3} / \mathrm{s}$ & $0,04 \mathrm{~m}^{3} / \mathrm{s}$ & $0,35 \mathrm{~m}^{3} / \mathrm{s}$ \\
\hline
\end{tabular}

Fonte: Elaboração própria com dados do CBH-PCJ, Fundação SEADE e consultas junto às empresas.

\section{ANÁLISE DAS VAZÕES NA BACIA DO RIO PIRACICABA}

Os dados fundamentais para o estudo das vazões foram extraídos dos postos fluviométricos monitorados pelo Departamento de Águas e Energia Elétrica do Estado de São Paulo (DAEE) e pelas Centrais Energéticas do Estado de São Paulo (CESP). Dentre os postos, por suas posições estratégicas, foram escolhidos os identificados na Tabela 06 e devidamente localizados na Figura 01.

O posto fluviométrico 4D-001 representa a sub-bacia do rio Jaguari e a 4D-009 a do Atibaia. O posto CESP representa a vazão do rio Piracicaba junto à cidade homônima, um dos pontos críticos a ser levado em consideração no presente estudo. Mesmo sem desconsiderar os dados disponíveis em outros postos da Tabela 06, os estudos foram concentrados fundamentalmente nessas três. Assim sendo, salvo se for explicitado, ao se fazer referência às sub-bacias do rio Jaguari e do rio Atibaia estão sendo consideradas as sub-bacias correspondentes aos postos 4D-001 e 4D-009, respectivamente. Da mesma maneira, quando se fizer referência à sub-bacia do Rio Piracicaba está sendo considerada a sub-bacia definida pelo posto CESP na cidade de Piracicaba e a confluência dos rios Atibaia e Jaguari.

Segundo a Tabela 7, a sub-bacia do rio Jaguari, incluindo a do rio Camanducaia, é a que apresenta maior descarga média, ou seja, $63 \mathrm{~m}^{3} / \mathrm{s}$, seguindo-se as sub-bacias do Piracicaba com $45 \mathrm{~m}^{3} / \mathrm{s}$, do Atibaia com 35 $\mathrm{m}^{3} / \mathrm{s}$ e do Corumbataí com $22 \mathrm{~m}^{3} / \mathrm{s}$. A descarga específica (vazão média por unidade de área da bacia) é também maior na do Jaguari, $4,6 \mathrm{~m}^{3} / \mathrm{ha}$.ano, sendo que as demais praticamente não se diferenciam, apresentando valores de 4,$0 ; 4,1$ e $3,9 \mathrm{~m}^{3} /$ ha.ano, respectivamente para as bacias do Corumbataí, Atibaia e Piracicaba. 
Tabela 06 - Identificação dos Postos Fluviométricos

\begin{tabular}{|c|c|c|c|c|c|c|}
\hline \multirow{2}{*}{$\begin{array}{c}\text { Rio / } \\
\text { Sub-Bacia }\end{array}$} & \multirow{2}{*}{$\begin{array}{c}\text { Prefixo } \\
\text { DAEE- } \\
\text { SP }\end{array}$} & \multirow{2}{*}{$\begin{array}{l}\text { Prefixo } \\
\text { DNAEE }\end{array}$} & \multirow{2}{*}{$\begin{array}{c}\text { Denominação } \\
\text { Estação }\end{array}$} & \multirow{2}{*}{$\begin{array}{c}\text { Área Bacia } \\
\text { Abrangida } \\
\left(\mathbf{k m}^{2}\right)\end{array}$} & \multicolumn{2}{|c|}{ Localização } \\
\hline & & & & & Latitude & Longitude \\
\hline \multirow{3}{*}{ Atibaia } & $3 \mathrm{D}-006$ & 62676000 & Bairro Ponte & 1918 & $22^{\circ} 58^{\prime}$ & $46^{\circ} 50^{\prime}$ \\
\hline & $3 \mathrm{D}-003$ & 62680000 & Des. Furtado & 2410 & $22^{\circ} 46^{\prime}$ & $46^{\circ} 59^{\prime}$ \\
\hline & 4D-009 & 62890000 & & 2682 & $22^{\circ} 45^{\prime}$ & $47^{\circ} 10^{\prime}$ \\
\hline Camanducaia & $3 \mathrm{D}-001$ & 6262800 & Faz. Barra & 928 & $22^{\circ} 40^{\prime}$ & $46^{\circ} 58^{\prime}$ \\
\hline \multirow{2}{*}{ Jaguari } & 3D-009 & 62605000 & Buenópolis & 1950 & $22^{\circ} 51^{\prime}$ & $46^{\circ} 47^{\prime}$ \\
\hline & $4 \mathrm{D}-001$ & 62632000 & Usina Ester & 3394 & $22^{\circ} 39^{\prime}$ & $47^{\circ} 13^{\prime}$ \\
\hline Corumbataí & $4 \mathrm{D}-021$ & 62709000 & Recreio & 1648 & $22^{\circ} 37^{\prime}$ & $47^{\circ} 41^{\prime}$ \\
\hline \multirow{3}{*}{ Piracicaba } & 4D-010 & 62695000 & Carioba & 7078 & $22^{\circ} 43^{\prime}$ & $47^{\circ} 21^{\prime}$ \\
\hline & $\operatorname{CESP}(*)$ & 62705000 & Piracicaba & 8500 & $22^{\circ} 41^{\prime}$ & $47^{\circ} 35^{\prime}$ \\
\hline & $4 \mathrm{D}-007$ & 62715000 & Artemis & 11040 & $22^{\circ} 41^{\prime}$ & $47^{\circ} 47^{\prime}$ \\
\hline
\end{tabular}

Vazões mínimas - A avaliação da disponibilidade hídrica toma por base a vazão mínima média de 7 dias e período de retorno de 10 anos $\left(\mathrm{Q}_{7,10}\right)$, e assume que o conjunto dos setores urbano, industrial e agrícola capta em toda a bacia do rio Piracicaba, à jusante do Sistema Cantareira, $26,7 \mathrm{~m}^{3} / \mathrm{s}$.

A disponibilidade hídrica média na bacia do Piracicaba é de $165 \mathrm{~m}^{3} / \mathrm{s}$ mas durante estiagens severas, cai para $40 \mathrm{~m}^{3} / \mathrm{s}$, para o que muito contribui a mencionada exportação de água para o abastecimento da RMSP à vazão média de $31 \mathrm{~m}^{3} / \mathrm{s}$.

Em meses mais secos, a disponibilidade hídrica na Bacia apresentou a vazão média de $128 \mathrm{~m}^{3} / \mathrm{s}$, podendo a vazão mínima chegar a $34 \mathrm{~m}^{3} / \mathrm{s}$. Deve-se ressaltar, entretanto, que a vazão disponível é superior a $50 \mathrm{~m}^{3} / \mathrm{s}$ em $95 \%$ do tempo e inferior a $34 \mathrm{~m}^{3} / \mathrm{s}$ somente em $5 \%$ do ano (SÃO PAULO, 1994).

Resulta que, para fins práticos, a disponibilidade de água na Bacia pode ser considerada algo entre $34 \mathrm{~m}^{3} / \mathrm{s}$ e $50 \mathrm{~m}^{3} / \mathrm{s}$ nos meses mais secos do ano, os quais coincidem com a época de maior consumo de água pela indústria e pela agropecuária.

Vazões médias - As Figuras 02, 03 e 04 apresentam os gráficos das vazões mensais mínimas, médias e máximas para os postos fluviométricos 4D-009, 4D-001 e CESP. As vazões médias e máximas foram calculadas para o período posterior ao ano de 1980, quando o Sistema Cantareira entrou em plena operação, e as vazões mínimas para todo o período em que existem dados disponíveis, de modo a identificar a menor vazão observada desde que a coleta de dados foi instituída. 
Figura 01 - Mapa da Bacia Hidrográfica do rio Piracicaba, com a localização dos corpos de água principais, postos fluviométricos, municípios e indústrias.

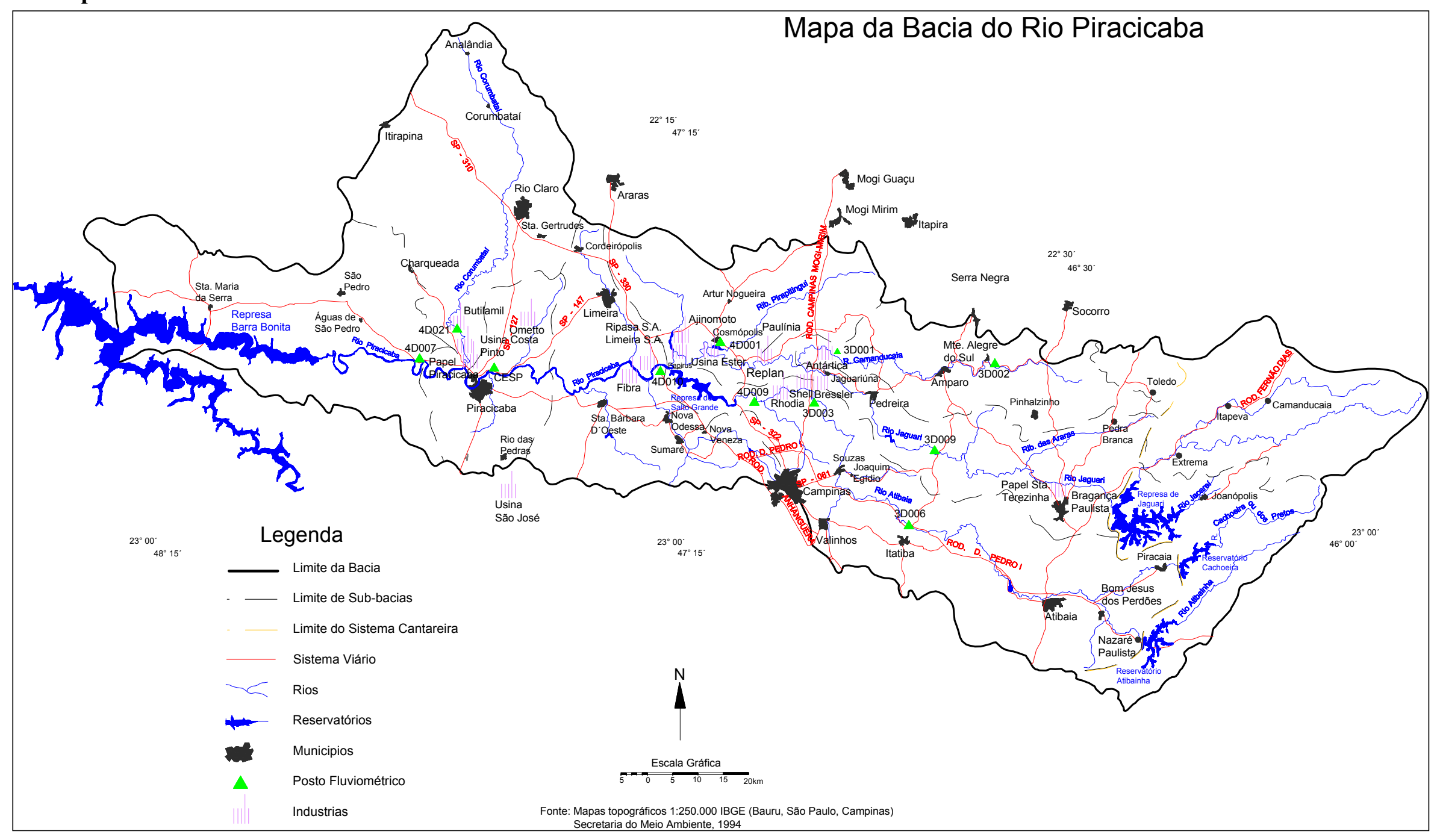




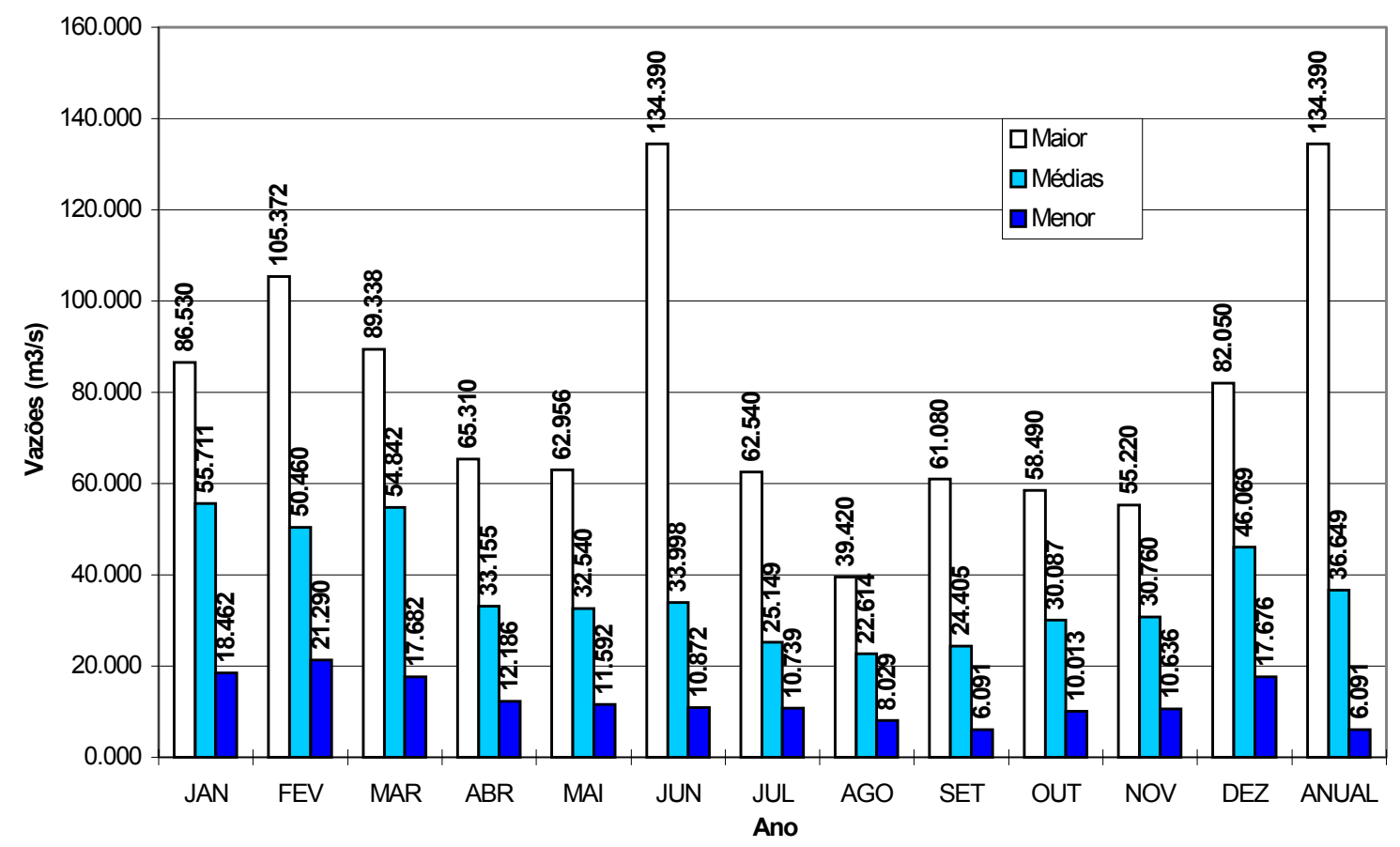

Figura 02 - Vazões médias mensais mínimas, médias e máximas para o Posto Fluviométrico 4D-009 - rio Atibaia (série histórica compreendendo o período de 1981 a 1996).

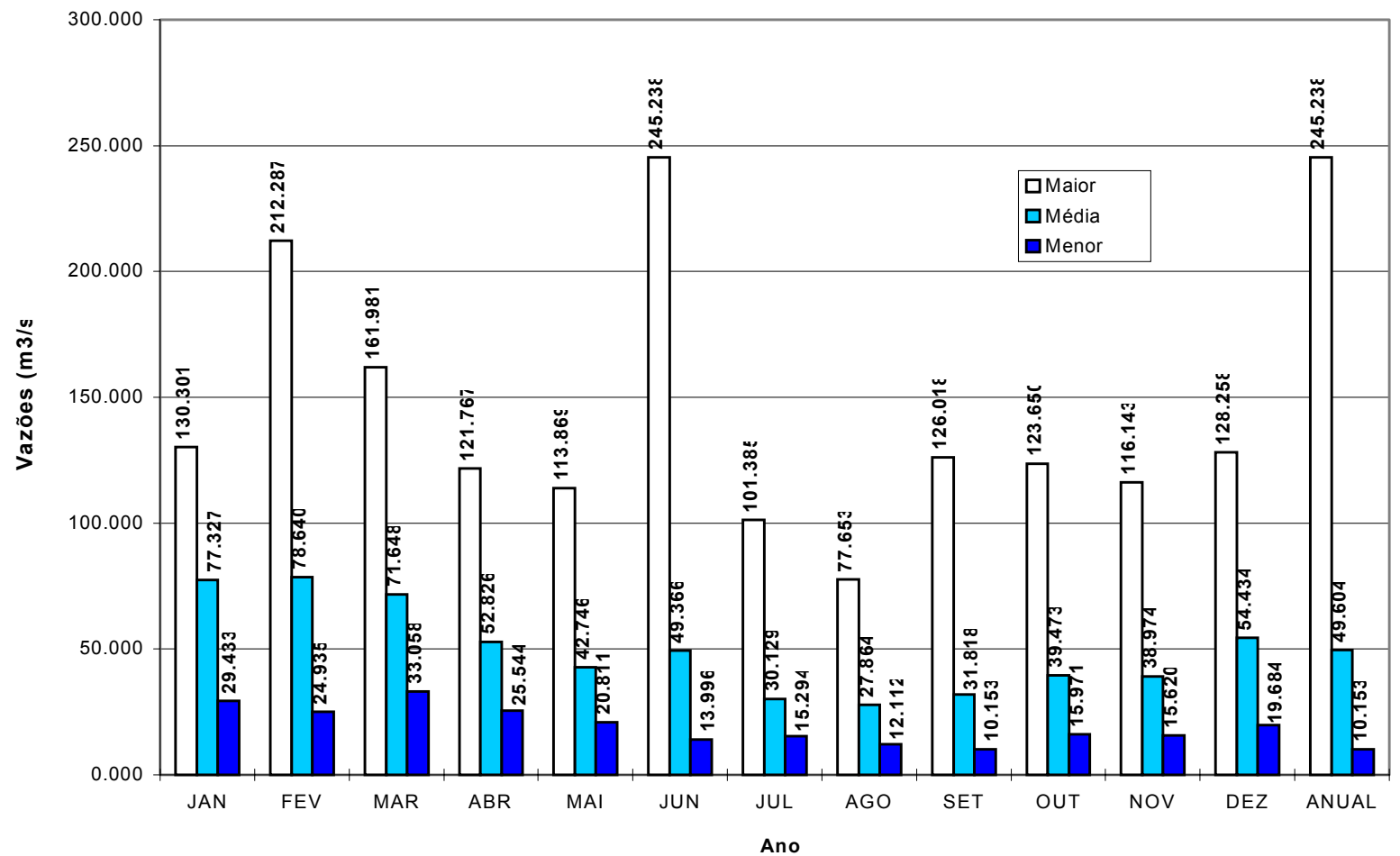

Figura 03- Vazões médias mensais mínimas, médias e máximas para o Posto Fluviométrico 4D-001 - rio Jaguari (série histórica compreendendo o período de 1981 a 1998). 


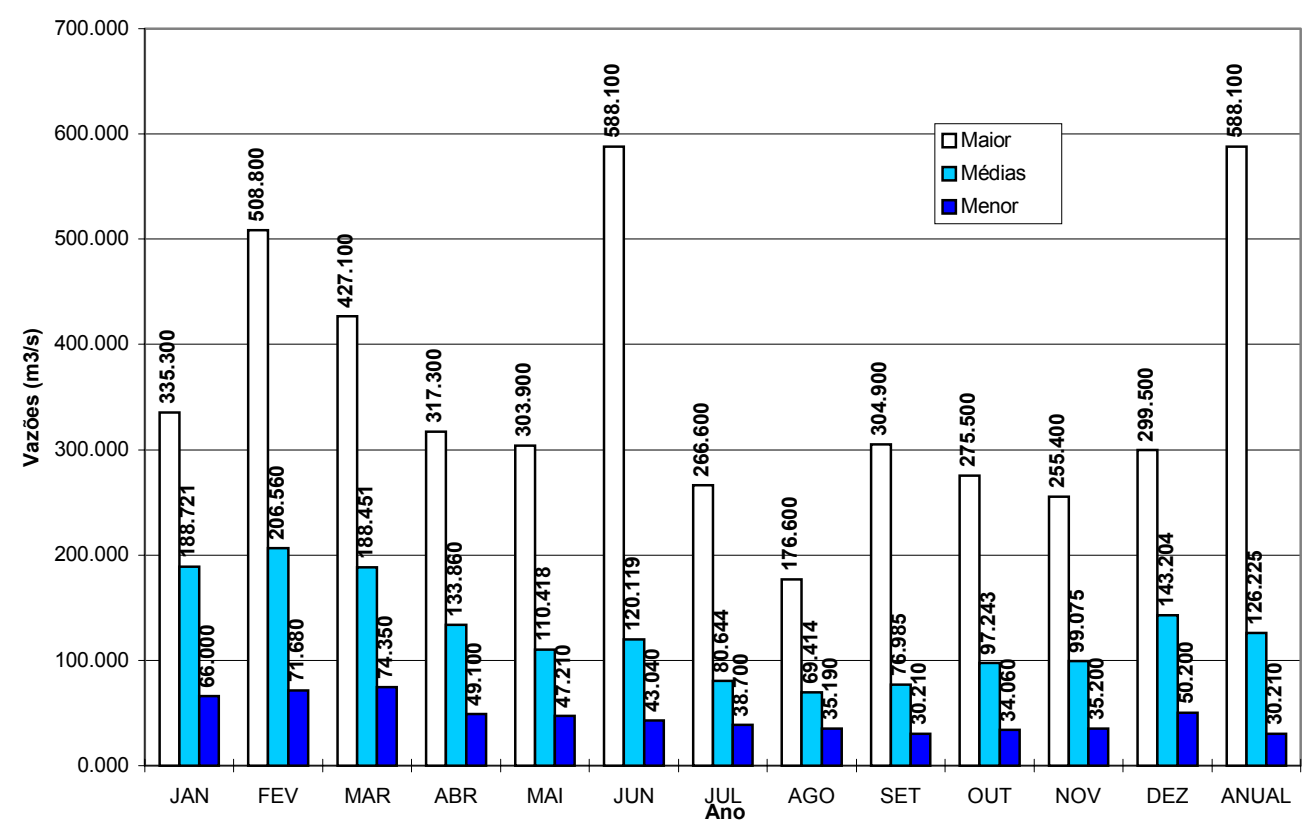

Figura 04 - Vazões médias mensais mínimas, médias e máximas para o Posto Fluviométrico CESP - Piracicaba (compreendendo o período de 1981 a 1995).

A Figura 04 apresenta as vazões para a estação CESP, saltando aos olhos que a operação do Sistema Cantareira não tem mantido a vazão mínima de $40 \mathrm{~m}^{3} / \mathrm{s}$ estabelecida para o rio Piracicaba junto à cidade homônima. As vazões médias mensais mínima minimorum são inferiores aos $40 \mathrm{~m}^{3} / \mathrm{s}$ de julho a novembro. Além disso, essas vazões médias mascaram valores diários bem abaixo da vazão mínima indicada na Figura, tais como $23 \mathrm{~m}^{3} / \mathrm{s}$ ocorridos em 1990. O mesmo ocorre no Atibaia, em Paulínia, onde valores de vazão tão baixos quanto $9 \mathrm{~m}^{3} / \mathrm{s}$ são apontados nesse mesmo ano (JAAKKO POYRY, 1992).

O balanço das vazões (Tabela 07) evidencia que a disponibilidade hídrica tanto na sub-bacia do rio Atibaia quanto na do Jaguari é crítica no período de estiagem, quando a vazão de demanda total $\mathrm{Q}_{\text {dem }}$ torna-se superior a vazão mínima média $Q_{7,10}$. Nessa situação não há disponibilidade hídrica em nenhuma dessas sub-bacias 
Tabela 07 - Síntese das Vazões e Demandas por Sub-Bacia (CBH/PCJ, 1996)

\begin{tabular}{|l|c|c|c|c|c|c|c|}
\hline Sub-Bacia & $\begin{array}{c}\text { Área } \\
\left(\mathbf{K m}^{2}\right)\end{array}$ & $\begin{array}{c}\mathbf{Q}_{\mathbf{7 1 0}} \\
\left(\mathbf{m}^{\mathbf{3}} / \mathbf{s}\right)\end{array}$ & $\begin{array}{c}\mathbf{Q}_{\mathbf{m e d}} \\
\left(\mathbf{m}^{\mathbf{3}} / \mathbf{s}\right)\end{array}$ & $\begin{array}{c}\mathbf{Q}_{\mathbf{9 5}} \\
\left(\mathbf{m}^{\mathbf{3}} / \mathbf{s}\right)\end{array}$ & $\begin{array}{c}\mathbf{Q}_{\text {bacia }} \\
\left(\mathbf{m}^{3} / \mathbf{s}\right)\end{array}$ & $\begin{array}{c}\mathbf{Q}_{\text {cant }} \\
\left(\mathbf{m}^{\mathbf{3}} / \mathbf{s}\right)\end{array}$ & $\begin{array}{c}\mathbf{Q}_{\text {dem }} \\
\left(\mathbf{m}^{3} / \mathbf{s}\right)\end{array}$ \\
\hline Rio Atibaia & 2760 & 10 & 35 & 15,2 & 8,84 & 11,4 & 20,2 \\
\hline Rio Jaguari & 4290 & 15 & 63 & 23 & 6,27 & 19,6 & 26,9 \\
\hline Rio Corumbataí & 1700 & 05 & 22 & 08 & 2,5 & 0 & 2,5 \\
\hline Rio Piracicaba & 3650 & 10 & 45 & 16 & 6,5 & 0 & 6,5 \\
\hline \multicolumn{1}{c|}{ Total } & 12400 & 40 & 165 & 62,2 & 24,11 & 31,0 & 56,1 \\
\hline
\end{tabular}

\section{Obs.:}

$\boldsymbol{Q}_{95}=$ vazão de estiagem com 95\% de permanência (igualada ou superada em 95\% do tempo);

$\boldsymbol{Q}_{\text {med }}=$ vazão média plurianual;

$\boldsymbol{Q}_{\text {cant }}=$ vazão retirada pelo Sistema Cantareira;

$\boldsymbol{Q}_{\text {bacia }}=$ demanda na bacia a jusante do Sistema Cantareira.

$\boldsymbol{Q}_{\text {dem }}=$ demanda total (urbana + industrial + irrigação + Sistema Cantareira) em cada subbacia

\section{CONCLUSÕES}

A vazão natural média da bacia do rio Piracicaba é de $186 \mathrm{~m}^{3} / \mathrm{s}$, os quais sustentam um volume de captação de $25 \mathrm{~m}^{3} / \mathrm{s}$ mais a transposição de águas pelo Sistema Cantareira para o abastecimento da Região Metropolitana de São Paulo à razão de $31 \mathrm{~m}^{3} / \mathrm{s}$. Intervenções de tal magnitude fazem com que nas estiagens mais severas a disponibilidade hídrica média caia para $40 \mathrm{~m}^{3} / \mathrm{s}$, com vazões mínimas médias mensais de até $34 \mathrm{~m}^{3} / \mathrm{s}$. De qualquer forma, a vazão média é superior a $50 \mathrm{~m}^{3} / \mathrm{s} \mathrm{em} 95 \%$ do ano e inferior a $34 \mathrm{~m}^{3} / \mathrm{s}$ somente em $5 \%$, o que permite considerar, para fins práticos, a disponibilidade de água na Bacia entre 34 e $50 \mathrm{~m}^{3} / \mathrm{s}$.

As tendências do crescimento da demanda de água na Bacia indicam a estabilização dos volumes de captação industrial, o crescimento do consumo urbano à taxa de $2 \%$ a.a. e do agropecuário em $1,1 \%$ a.a. Percentuais que sinalizam um aumento de demanda total de aproximadamente $0,35 \mathrm{~m}^{3} / \mathrm{s}$ a.a..

O balanço das vazões superficiais indica que a sub-bacia do rio Jaguari, as maiores vazões disponíveis, de $23,4 \mathrm{~m}^{3} / \mathrm{s}$. Em apenas $13 \%$ do ano essa Sub-Bacia não dispõe, considerando-se a necessidade de sua contribuição para manter a vazão de $40 \mathrm{~m}^{3} / \mathrm{s}$ no rio Piracicaba junto à cidade homônima, de vazões para atender a demanda prevista. De acordo com as informações de Lopes (1994), avalia-se que os Sistemas Aqǘferos Cristalino (Reserva explotável - 9,68 $\mathrm{m}^{3} / \mathrm{s}$ ), Tubarão - Aqüífero Itararé (Reserva explotável - 3,35 $\mathrm{m}^{3} / \mathrm{s}$ ); Diabásio (Reserva explotável - 0,75 $\mathrm{m}^{3} / \mathrm{s}$ ) e Cenozóico, possuem uma limitada reserva de água subterrânea, porém 
suficientes para abastecimento público em bairros. A situação de explotação das águas na região já evidencia problemas e conflitos podendo identificar áreas na Região Metropolitana de Campinas, já com déficit de disponibilidade hídrica (YOSHINAGA-PEREIRA, 1997). Os estudos hidrogeológicos também evidenciam o uso comum dessas águas por praticamente todas as indústrias, condomínios e comércios em geral e um boom no crescimento do uso deste recurso nas últimas décadas.

Por fim, o trabalho apresenta as dificuldades de abastecimento de água enfrentadas atualmente por municípios da bacia do Piracicaba e apontam para a necessidade de atuação conjunta do poder público e da iniciativa privada para o equacionamento do problema da utilização dos recursos hídricos na região, de modo que a solução do problema de um resulte na superação das dificuldades enfrentadas pelo outro.

\section{REFERÊNCIAS}

COMITÊ DAS BACIAS DOS RIOS PIRACICABA, CAPIVARI E JUNDIAÍ (CBH-PCJ). Relatório de Situação dos Recursos Hídricos 1995. São Paulo. DAEE.. 65p., 1996.

COMITÊ DAS BACIAS DOS RIOS PIRACICABA, CAPIVARI E JUNDIAÍ (CBH-PCJ). Relatório de Situação dos Recursos Hídricos 1993. Versão Preliminar aprovada na $2^{a}$ Reunião do CBH-PCJ em 15/04/94. 99p., 1994.

GUEDA, O ; STURION, A C.; CARON, D. Evolução da Agropecuária na Bacia do Rio Piracicaba e o Consumo de Água. SEADE. Relatório Final.. vol.1, 51p., 1992.

HIDROPLAN/DAEE. COPLASA, ETEP, Figueiredo Ferraz, Hidroconsult.

Plano Integrado de Aproveitamento e Controle dos Recursos Hídricos das bacias Alto Tietê, Piracicaba e Baixada Santista. CDROM. 1994.

JAAKKO POYRY ENGENHARIA LTDA. Plano Diretor de Captação e Produção de Água para Abastecimento Público nas Bacias dos rios Piracicaba e Capivari. Convênio Intermunicipal das Bacias dos rios Piracicaba e Capivari - Departamento de Água e Energia Elétrica. São Paulo. 1992.

LOPES, M.F.C. Condições de ocorrência de água subterrânea nas

Bacias dos Rios Piracicaba e Capivari. Dissertação de Mestrado FEC/UNICAMP. . 83p., 1994.

MONTICELI, J.J.; MARTINS, J.P.S. A luta pela água - Nas Bacias dos

Rios Piracicaba e Capivari - SP. Editora EME. $1^{\text {a }}$ edição. . 126p., 1993. 
PELLEGRINO, G.Q. Análise espaço temporal de dados hidrológicos da bacia do rio Piracicaba. Dissertação de Mestrado. Escola Superior de Agricultura Luiz de Queiroz, USP - Piracicaba. 117 pp., 1995.

SEADE, FUNDAÇÃO. http://www.seade.org.br. Dados populacionais referentes a população na Região de Campinas. 1998.

SÃO PAULO (Estado). Secretaria do Meio Ambiente (SMA). Estabelecimento de metas ambientais e reenquadramento dos corpos de água: Bacia do Rio Piracicaba. SMA. São Paulo. 81p., 1994. (Série Relatórios).

YOSHINAGA-PEREIRA, S. Proposta de representação cartográfica na avaliação hidrogeológica para o estudo de planejamento e meio ambiente, exemplo da Região Metropolitana de Campinas - SP. Tese de Doutoramento IG/USP. 190p.,1996. 